\title{
Can the duration of tuberculosis treatment be shortened with higher dosages of rifampicin?
}

\author{
Noton K. Dutta ${ }^{1 *}$ and Petros C. Karakousis ${ }^{1,2}$ \\ 1 Department of Medicine, Center for Tuberculosis Research, Johns Hopkins University School of Medicine, Baltimore, MD, \\ USA, ${ }^{2}$ Department of International Health, Johns Hopkins Bloomberg School of Public Health, Baltimore, MD, USA
}

Keywords: Mycobacterium tuberculosis, rifampicin, treatment improvement, persistence, reactivation, animal models, clinical trial

\section{INTRODUCTION}

Strategies involving new applications of existing drugs are urgently needed to reduce the time required to cure patients with drug-susceptible and drug-resistant tuberculosis (TB). Use of high-dosage rifampicin is one such approach. Recent data from preclinical animal models (Hu et al., 2015) and clinical studies (Boeree et al., 2015) support a potential role for highdosage rifampicin in TB chemotherapy, although more studies are required to guide optimal

OPEN ACCESS

Edited by:

Tzi Bun Ng,

The Chinese University of Hong Kong

China

Reviewed by:

Fabian Cieplik,

University Medical Center

Regensburg, Germany

Xian-Zhi Li,

Health Canada, Canada

${ }^{*}$ Correspondence:

Noton K. Dutta

ndutta1@jhmi.edu

Specialty section:

This article was submitted to

Antimicrobials, Resistance and

Chemotherapy,

a section of the journal

Frontiers in Microbiology

Received: 29 June 2015 Accepted: 28 September 2015

Published: 14 October 2015

Citation:

Dutta NK and Karakousis PC (2015)

Can the duration of tuberculosis treatment be shortened with higher

dosages of rifampicin?

Front. Microbiol. 6:1117.

doi: 10.3389/fmicb.2015.01117 clinical management. Specifically, further basic preclinical research is needed to: (i) Develop pharmacokinetic/pharmacodynamic models to improve our understanding of drug bioavailability and activity in tissues; (ii) Determine the antimicrobial efficacy and the ability of high-dosage rifampicin to reduce the emergence of antibiotic resistance; and (iii) Explore high-dosage rifampicin for evaluation of new combination regimens to achieve the ultimate goal of shortening TB treatment and achieving stable cure without relapse.

\section{AN UPDATE ON THE USE OF HIGH-DOSAGE RIFAMPICIN FOR THE TREATMENT OF TUBERCULOSIS}

\section{Rifampicin and its Role in Dots (Directly Observed Treatment, Short-course)}

Combination chemotherapy has been the standard of care for TB since the 1950s, when it was demonstrated that combining streptomycin with para-aminosalicylic acid and, later, with isoniazid prevented the emergence of drug resistance and enabled reliable cures following 18-24 months of treatment (1972). In the $1980 \mathrm{~s}$, the sequential incorporation of rifampicin and pyrazinamide accelerated the eradication of bacterial "persisters" and shortened the duration of treatment needed to prevent relapse. Combining the synergistic antimicrobial properties of rifampicin and pyrazinamide with the potent bactericidal activity of isoniazid formed the basis of the current 6-month "short-course" regimen, which showed the least frequency of relapse. Yet, despite the high efficacy of this treatment and efforts to implement it throughout the world, TB remains a global health emergency, in part because even a 6-month regimen poses formidable challenges for the resource-limited healthcare infrastructures of many TB-endemic countries.

\section{Mechanism of Action and Resistance}

Rifampicin is a semisynthetic derivative of rifamycin B which is produced by Amycolatopsis (formerly Streptomyces) rifamycinica. It is one of the key drugs for the short course TB regimen and possesses bactericidal as well as sterilizing activity against tubercle bacilli in both cellular and 
extracellular locations. The rifamycins are highly proteinbound in plasma, but easily diffuse across the Mycobacterium tuberculosis cell envelope due to their lipophilic nature (Wade and Zhang, 2004). The bactericidal activity of the rifamycins is attributed to their ability to inhibit transcription by binding with high affinity to bacterial DNA-dependent RNA polymerase (Hartmann et al., 1967; Jin and Gross, 1991; Campbell et al., 2001).

The development of rifampicin resistance is strongly associated with lower serum drug concentrations (Pasipanodya et al., 2013), while higher maximum concentration $\left(C_{\max }\right)$ and area under the serum concentration-time curve $\left(\mathrm{AUC}_{0-24}\right)$ inhibit the development of rifampicin resistance (Gumbo et al., 2007). Mutations in the $r p o B$ gene account for over $95 \%$ of clinical cases of rifampicin resistance and are commonly associated with the presence of multidrug-resistant tuberculosis (Shah et al., 2007). Unlike mutations in codons 531 and 526, which confer high-level resistance to rifampicin (MIC > $32 \mu \mathrm{g} / \mathrm{ml}$ ) and cross-resistance to all rifamycins (Wade and Zhang, 2004), mutations in codons 511, 516, and 522 are associated with low- or high-level resistance to rifampicin (MIC 2-32 $\mu \mathrm{g} / \mathrm{ml}$ ) (Bodmer et al., 1995; Moghazeh et al., 1996; Williams et al., 1998).

Consistent with the clinical data, selection of spontaneous rifampicin resistance in vitro in the $M$. tuberculosis laboratory reference strain $\mathrm{H} 37 \mathrm{Rv}$ indicate that the Ser $_{531}$-to-Leu mutation and multiple mutations in codon 526 occur at a significantly higher frequency than other point mutations (Billington et al., 1999). In fact, mutants with low-level rifampicin resistance appear to be better adapted to in vivo growth than mutants with high-level rifampicin resistance. Mutant strains can be enriched in the presence of drug pressure (Mariam et al., 2004). Whether higher dosages of rifampicin facilitate the emergence of mutants with higher MIC of the drug requires further study. Louw et al. showed that the level of rifampicin resistance is determined by the activation of efflux and transporter genes (Louw et al., 2011). Recent data suggest that in addition to classical mutations in rpoB, the efflux pumps Rv2333, DrrB, DrrC, Rv0842, BacA, and EfpA may have a role in rifampicin resistance (Li et al., 2015).

\section{Pharmacokinetics and Pharmacodynamics}

Rifampicin is used at a dose of $600 \mathrm{mg}$ per day throughout the 6-month TB treatment course. This dosing scheme was determined in the $1960 \mathrm{~s}$ based on cost and efficacy, although the highest tolerable dose was not defined (Steingart et al., 2011; van Ingen et al., 2011). Rifampicin's microbial killing was linked to the area under the $\mathrm{AUC}_{0-24} /$ minimum inhibitory concentration (MIC) ratio (Jayaram et al., 2003). However, many patients achieve rifampicin $\mathrm{AUC}_{0-24} / \mathrm{MIC}$ and $\mathrm{C}_{\max } / \mathrm{MIC}$ ratios associated with suboptimal microbial killing and resistance suppression (Peloquin et al., 1997), indicating that higher dosages of rifampicin could improve treatment outcomes, so long as patients can tolerate them. Following delivery of the standard 600-mg dosage, rifampicin concentrations attained at the site of infection were determined to be too low (Ziglam et al., 2002; Goutelle et al., 2009). Interestingly, the use of a 1200$\mathrm{mg}$ rifampicin dosage significantly increased the probability of attaining $\mathrm{AUC}_{0-24} / \mathrm{MIC}$ or $\mathrm{C}_{\max } / \mathrm{MIC}$ ratios compatible with bacterial killing (Goutelle et al., 2009). Recent studies indicate that increases in rifampicin dosage result in concentrations that are more than dose-proportional; specifically, a 2-fold increase in dosage from 10 to $20 \mathrm{mg} / \mathrm{kg}$ daily results in a 4 -fold increase in the $\mathrm{AUC}_{0-24}$ and enhanced early bactericidal activity for each increase in dosage (Boeree et al., 2015). In addition, higher dosages of rifampicin may result in nonlinear increases in drug concentrations inside the bacteria, a phenomenon possibly related to saturation of bacterial efflux pumps (Gumbo et al., 2007). Piddock et al. demonstrated the accumulation of rifampicin by mycobacteria in the presence of efflux inhibitor reserpine (Piddock et al., 2000). There is a need to assess whether high-dosage rifampicin affects the pharmacokinetics of other anti-TB drugs and antiretroviral drugs, particularly its inductive effect on the cytochrome P450 enzyme system. While physiologically based pharmacokinetic modeling is often motivated by animal-to-human scaling (Savic et al., 2014), the differences in variability, absorption, distribution, metabolism, and excretion of rifampicin between mice and humans should be considered (Lyons et al., 2013). For example, penetration of rifampicin into lung cavities cannot be modeled in standard mouse models of TB, which lack cavities (Lenaerts et al., 2015). The rate of intestinal absorption of rifampicin is reduced in mice when given at higher dosages, although this does not affect total AUC. The impact of higher protein binding in mice (96\%) relative to human (89\%) on differences in pharmacokinetic and pharmacodynamic properties of the drugs is unknown. In addition, rifampicin can reduce the plasma concentrations of drugs that are not metabolized (e.g., digoxin) by inducing drug transporters such as P-glycoprotein. Finally, mice do not generate 25-desacetyl rifampicin, which is the main metabolite of rifampicin in humans (Wilkins et al., 2008; Dutta et al., 2012, 2013).

\section{Recent Advances}

Recently, existing drugs are being repurposed or optimized for $\mathrm{TB}$ with the goal of shortening the duration of treatment for drug-sensitive and drug-resistant TB. Use of high-dosage rifampicin is one such approach. Results from studies with mice (Jayaram et al., 2003) and early bactericidal activity studies (Diacon et al., 2007) indicate that a single dosage of $600 \mathrm{mg}$ of rifampicin in $\mathrm{TB}$ treatment is at the lower end of the concentration-response curve. Using the murine TB model, Rosenthal et al. showed that increasing the dosage of rifampicin significantly increased the sterilizing activity of the regimen (Rosenthal et al., 2012). Steenwinkel et al. reported that an eightfold increase in the currently used $10 \mathrm{mg} / \mathrm{kg}$ rifampin dosage was well tolerated and allowed reduction of therapy duration from 6 to 2 months (de Steenwinkel et al., 2013). The study by $\mathrm{Hu}$ et al. (2015) investigated the role of high-dosage rifampicin against Mycobacterium tuberculosis persisters in an in vitro model of progressive hypoxia and in the Cornell mouse model of persistence. The authors found a dose-proportional increase in rifampicin $\mathrm{C}_{\max }$ and $\mathrm{AUC}_{0-24}$, resulting in eradication of resuscitation promoting factor-dependent persisters. $\mathrm{Hu}$ et al. study also showed that lung culture-conversion and relapse-free 
cure were obtained much earlier in mice treated with highdosage rifampicin $(50 \mathrm{mg} / \mathrm{kg}$ ) (Hu et al., 2015), thereby allowing for an abbreviated treatment course without incurring disease relapse. It has been poignantly recognized in the TB field recently that observations made in mice are not necessarily predictive of outcomes in human clinical trials of TB chemotherapy, nor is early “sterilization" a predictor of cure (Gillespie et al., 2014).

Recently, data from patients with osteoarticular tuberculosis suggest that increasing the rifampicin concentration at the site of infection may optimize this drug's antitubercular effect, even against some rifampicin-resistant isolates, if systemic toxicity can be minimized (Zhang et al., 2014). Historical trials suggest that higher than standard rifampicin dosing results in improved culture conversion rates (please see systematic review (Steingart et al., 2011). Currently, several clinical trials are examining the efficacy and safety of higher dosages of rifampicin than the currently used dosage of $10 \mathrm{mg} / \mathrm{kg}$ against drug-susceptible TB. Phase II and III clinical trials evaluating higher dosages of rifampicin and other rifamycins are needed to confirm efficacy and assure tolerability. PanACEA (the Pan-African Consortium for the Evaluation of Antituberculosis Antibiotics) which is funded by the European and Developing Countries Clinical Trials Partnership, has found that administering up to $35 \mathrm{mg} / \mathrm{kg}$ of rifampicin is safe and well tolerated, resulting in a non-linear increase in exposure to rifampicin without an apparent ceiling effect, and increased early bactericidal activity at 14 days (HIGHRIF1-phase IIA, multiple dose rising study grouping 20, 25, 30 up to $35 \mathrm{mg} \mathrm{RIF} / \mathrm{kg}$, NCT01392911); (Boeree et al., 2015). However, it is important to note that as we are dealing with months of exposure to rifampin, a drug administration of 2 weeks only qualifies as an acute toxicity study and cannot provide sufficient estimation of toxicities from subacute or chronic exposure. A second trial (HIGHRIF2- phase IIb, NCT00760149) is examining the efficacy of rifampicin given at 10,15 , and $20 \mathrm{mg} / \mathrm{kg}$ daily. Although the microbiological data are not yet available, this study found no serious adverse events for 2 months of rifampicin at 15 and $20 \mathrm{mg} / \mathrm{kg}$. HIGHRIF3 (phase II) is a dosage-ranging study designed to identify the optimal rifampicin dosage for evaluation of efficacy. The group recently started the above-described multi-arm multi-stage study, which tests one group with $35 \mathrm{mg} / \mathrm{kg}$ of rifampicin (isoniazid/rifampin 35 /pyrazinamide/ethambutol), a second group with $20 \mathrm{mg} / \mathrm{kg}$ of rifampicin combined with moxifloxacin (isoniazid/rifampicin 20 /pyrazinamide/moxifloxacin), and a third with $20 \mathrm{mg} / \mathrm{kg}$ of rifampicin combined with the novel ethylenediamine, SQ109. Preliminary analysis of the data suggests that the first two groups may shorten the duration of TB treatment. The International Consortium for Trials of Chemotherapeutic Agents in Tuberculosis (INTERTB) will soon publish the results of the RIFATOX study, which indicated that

\section{REFERENCES}

(1972). Controlled clinical trial of short-course (6-month) regimens of chemotherapy for treatment of pulmonary tuberculosis. Lancet 1, 1079-1085. rifampicin at 900 and $1200 \mathrm{mg}$ daily for the first 4 months of the standard 6-month regimen was safe, with no increase in serious adverse events (ISRCTN55670677). However, these higher dosages of rifampicin did not significantly improve culture conversion rates at 2 months. Based on these results, a phase III study (RIFASHORT) has been initiated to assess the treatmentshortening potential of high-dosage (1200/1800 mg) rifampicin ( 2 months of isoniazid/rifampicin/pyrazinamide/ethumbutol + 4 months of isoniazid/rifampicin). The NIAID HIRIF study, started in September 2013, is a randomized trial of highdosage rifampicin in patients with new, smear-positive TB (NCT01408914). French National Institute for Health and Medical Research-French National Agency for Research on AIDS and Viral Hepatitis (Inserm-ANRS) started RIFAVIRENZ (NCT01986543), a drug-drug interaction study between high dosage rifampicin and efavirenz in the context of pulmonary tuberculosis and HIV co-infection. Recent report suggests that rifampicin and rifapentine significantly reduce concentrations of bedaquiline, a new anti-TB drug (Svensson et al., 2015).

\section{CONCLUSIONS}

Use of high-dosage rifampicin against Mycobacterium tuberculosis is promising as it may not only result in enhanced killing of mycobacteria and shorter therapy duration, but may also result in prevention of drug resistance (Gumbo et al., 2007; Goutelle et al., 2009; Rosenthal et al., 2012; de Steenwinkel et al., 2013; Boeree et al., 2015; Hu et al., 2015), which are highly desirable properties in an anti-TB regimen. While these preclinical studies are subject to questions regarding their predictive accuracy for assessing the efficacy of anti-TB regimens, the results of ongoing phase IIB studies promise to provide further guidance on the optimal dosage of rifampin (Boeree et al., 2015). However, whether high-dosage rifampicin results in reduced relapse rates remains to be explored in clinical studies. Additional preclinical research using wellvalidated animal models of tuberculosis is warranted to guide the future study of high-dosage rifampicin in clinical trials. For joint HIV-TB treatment, it is important to determine if high-dosage rifampicin can shorten the time required to cure $\mathrm{TB}$ without increasing adverse events and drug interactions with other antitubercular drugs and antiretroviral agents.

\section{FUNDING}

This study was funded in part by National Institutes of Health grant R01AI106613 (to PK) and by the Johns Hopkins University Center for AIDS Research Tangible Research Initiative award (to ND). 
Bodmer, T., Zürcher, G., Imboden, P., and Telenti, A. (1995). Mutation position and type of substitution in the beta-subunit of the RNA polymerase influence in vitro activity of rifamycins in rifampicin-resistant Mycobacterium tuberculosis. J. Antimicrob. Chemother. 35, 345-348. doi: 10.1093/jac/35.2.345

Boeree, M. J., Diacon, A. H., Dawson, R., Narunsky, K., du Bois, J., Venter, A., et al. (2015). A dose-ranging trial to optimize the dose of rifampin in the treatment of tuberculosis. Am. J. Respir. Crit. Care Med. 191, 1058-1065. doi: 10.1164/rccm.201407-1264OC

Campbell, E. A., Korzheva, N., Mustaev, A., Murakami, K., Nair, S., Goldfarb, A., et al. (2001). Structural mechanism for rifampicin inhibition of bacterial rna polymerase. Cell 104, 901-912. doi: 10.1016/S0092-8674(01)00286-0

de Steenwinkel, J. E., Aarnoutse, R. E., de Knegt, G. J., ten Kate, M. T., Teulen, M., Verbrugh, H. A., et al. (2013). Optimization of the rifampin dosage to improve the therapeutic efficacy in tuberculosis treatment using a murine model. Am. J. Respir. Crit. Care Med. 187, 1127-1134. doi: 10.1164/rccm.201207-1210OC

Diacon, A. H., Patientia, R. F., Venter, A., van Helden, P. D., Smith, P. J., McIlleron, H., et al. (2007). Early bactericidal activity of high-dose rifampin in patients with pulmonary tuberculosis evidenced by positive sputum smears. Antimicrob. Agents Chemother. 51, 2994-2996. doi: 10.1128/AAC.01474-06

Dutta, N. K., Alsultan, A., Peloquin, C. A., and Karakousis, P. C. (2013). Preliminary pharmacokinetic study of repeated doses of rifampin and rifapentine in guinea pigs. Antimicrob. Agents Chemother. 57, 1535-1537. doi: 10.1128/AAC.01933-12

Dutta, N. K., Illei, P. B., Peloquin, C. A., Pinn, M. L., Mdluli, K. E., Nuermberger, E. L., et al. (2012). Rifapentine is not more active than rifampin against chronic tuberculosis in guinea pigs. Antimicrob. Agents Chemother. 56, 3726-3731. doi: 10.1128/AAC.00500-12

Gillespie, S. H., Crook, A. M., McHugh, T. D., Mendel, C. M., Meredith, S. K., Murray, S. R., et al. (2014). Four-month moxifloxacin-based regimens for drug-sensitive tuberculosis. N. Engl. J. Med. 371, 1577-1587. doi: 10.1056/NEJMoa1407426

Goutelle, S., Bourguignon, L., Maire, P. H., van Guilder, M., Conte, J. E. Jr., and Jelliffe, R. W. (2009). Population modeling and Monte Carlo simulation study of the pharmacokinetics and antituberculosis pharmacodynamics of rifampin in lungs. Antimicrob. Agents Chemother. 53, 2974-2981. doi: 10.1128/AAC.01520-08

Gumbo, T., Louie, A., Deziel, M. R., Liu, W., Parsons, L. M., Salfinger, M., et al. (2007). Concentration-dependent Mycobacterium tuberculosis killing and prevention of resistance by rifampin. Antimicrob. Agents Chemother. 51, 3781-3788. doi: 10.1128/AAC.01533-06

Hartmann, G., Honikel, K. O., Knüsel, F., and Nüesch, J. (1967). The specific inhibition of the DNA-directed RNA synthesis by rifamycin. Biochim. Biophys. Acta 145, 843-844. doi: 10.1016/0005-2787(67)90147-5

Hu, Y., Liu, A., Ortega-Muro, F., Alameda-Martin, L., Mitchison, D., and Coates, A. (2015). High-dose rifampicin kills persisters, shortens treatment duration, and reduces relapse rate in vitro and in vivo. Front. Microbiol. 6:641. doi: 10.3389/fmicb.2015.00641

Jayaram, R., Gaonkar, S., Kaur, P., Suresh, B. L., Mahesh, B. N., Jayashree, R., et al. (2003). Pharmacokinetics-pharmacodynamics of rifampin in an aerosol infection model of tuberculosis. Antimicrob. Agents Chemother. 47, 2118-2124. doi: 10.1128/AAC.47.7.2118-2124.2003

Jin, D. J., and Gross, C. A. (1991). RpoB8, a rifampicin-resistant terminationproficient RNA polymerase, has an increased $\mathrm{Km}$ for purine nucleotides during transcription elongation. J. Biol. Chem. 266, 14478-14485.

Lenaerts, A., Barry, C. E. III, and Dartois, V. (2015). Heterogeneity in tuberculosis pathology, microenvironments and therapeutic responses. Immunol. Rev. 264, 288-307. doi: 10.1111/imr.12252

Li, G., Zhang, J., Guo, Q., Wei, J., Jiang, Y., Zhao, X., et al. (2015). Study of efflux pump gene expression in rifampicin-monoresistant Mycobacterium tuberculosis clinical isolates. J. Antibiot. 68, 431-435. doi: 10.1038/ja.2015.9

Louw, G. E., Warren, R. M., Gey van Pittius, N. C., Leon, R., Jimenez, A., Hernandez-Pando, R., et al. (2011). Rifampicin reduces susceptibility to ofloxacin in rifampicin-resistant Mycobacterium tuberculosis through efflux. Am. J. Respir. Crit. Care Med. 184, 269-276. doi: 10.1164/rccm.201011-1924OC

Lyons, M. A., Reisfeld, B., Yang, R. S., and Lenaerts, A. J. (2013). A physiologically based pharmacokinetic model of rifampin in mice. Antimicrob. Agents Chemother. 57, 1763-1771. doi: 10.1128/AAC.01567-12

Mariam, D. H., Mengistu, Y., Hoffner, S. E., and Andersson, D. I. (2004). Effect of rpoB mutations conferring rifampin resistance on fitness of
Mycobacterium tuberculosis. Antimicrob. Agents Chemother. 48, 1289-1294. doi: 10.1128/AAC.48.4.1289-1294.2004

Moghazeh, S. L., Pan, X., Arain, T., Stover, C. K., Musser, J. M., and Kreiswirth, B. N. (1996). Comparative antimycobacterial activities of rifampin, rifapentine, and KRM-1648 against a collection of rifampin-resistant Mycobacterium tuberculosis isolates with known rpoB mutations. Antimicrob. Agents Chemother. 40, 2655-2657.

Pasipanodya, J. G., McIlleron, H., Burger, A., Wash, P. A., Smith, P., and Gumbo, T. (2013). Serum drug concentrations predictive of pulmonary tuberculosis outcomes. J. Infect. Dis. 208, 1464-1473. doi: 10.1093/infdis/jit352

Peloquin, C. A., Jaresko, G. S., Yong, C. L., Keung, A. C., Bulpitt, A. E., and Jelliffe, R. W. (1997). Population pharmacokinetic modeling of isoniazid, rifampin, and pyrazinamide. Antimicrob. Agents Chemother. 41, 2670-2679.

Piddock, L. J., Williams, K. J., and Ricci, V. (2000). Accumulation of rifampicin by Mycobacterium aurum, Mycobacterium smegmatis and Mycobacterium tuberculosis. J. Antimicrob. Chemother. 45, 159-165. doi: 10.1093/jac/45.2.159

Rosenthal, I. M., Tasneen, R., Peloquin, C. A., Zhang, M., Almeida, D., Mdluli, K. E., et al. (2012). Dose-ranging comparison of rifampin and rifapentine in two pathologically distinct murine models of tuberculosis. Antimicrob. Agents Chemother. 56, 4331-4340. doi: 10.1128/AAC.00912-12

Savic, R. M., Lu, Y., Bliven-Sizemore, E., Weiner, M., Nuermberger, E., Burman, W., et al. (2014). Population pharmacokinetics of rifapentine and desacetyl rifapentine in healthy volunteers: nonlinearities in clearance and bioavailability. Antimicrob. Agents Chemother. 58, 3035-3042. doi: 10.1128/AAC.01918-13

Shah, N. S., Wright, A., Bai, G. H., Barrera, L., Boulahbal, F., Martín-Casabona, N., et al. (2007). Worldwide emergence of extensively drug-resistant tuberculosis. Emerging Infect. Dis. 13, 380-387. doi: 10.3201/eid1303.061400

Steingart, K. R., Jotblad, S., Robsky, K., Deck, D., Hopewell, P. C., Huang, D., et al. (2011). Higher-dose rifampin for the treatment of pulmonary tuberculosis: a systematic review. Int. J. Tuberc. Lung Dis. 15, 305-316.

Svensson, E. M., Murray, S., Karlsson, M. O., and Dooley, K. E. (2015). Rifampicin and rifapentine significantly reduce concentrations of bedaquiline, a new antiTB drug. J. Antimicrob. Chemother. 70, 1106-1114. doi: 10.1093/jac/dku504

van Ingen, J., Aarnoutse, R. E., Donald, P. R., Diacon, A. H., Dawson, R., Plemper van Balen, G., et al. (2011). Why do we use $600 \mathrm{mg}$ of Rifampicin in tuberculosis treatment? Clin. Infect. Dis. 52, e194-e199. doi: 10.1093/cid/cir184

Wade, M. M., and Zhang, Y. (2004). Mechanisms of drug resistance in Mycobacterium tuberculosis. Front. Biosci. 9, 975-994. doi: 10.274 $1 / 1289$

Wilkins, J. J., Savic, R. M., Karlsson, M. O., Langdon, G., McIlleron, H., Pillai, G., et al. (2008). Population pharmacokinetics of rifampin in pulmonary tuberculosis patients, including a semimechanistic model to describe variable absorption. Antimicrob. Agents Chemother. 52, 2138-2148. doi: 10.1128/AAC.00461-07

Williams, D. L., Spring, L., Collins, L., Miller, L. P., Heifets, L. B., Gangadharam, P. R., et al. (1998). Contribution of rpoB mutations to development of rifamycin cross-resistance in Mycobacterium tuberculosis. Antimicrob. Agents Chemother. $42,1853-1857$.

Zhang, Z., Dai, F., Luo, F., Zhong, M., Huang, Z., Hou, T., et al. (2014). Could highconcentration rifampicin kill rifampicin-resistant $M$. tuberculosis ? Rifampicin MIC test in rifampicin-resistant isolates from patients with osteoarticular tuberculosis. J. Orthop. Surg. Res. 9, 124. doi: 10.1186/s13018-0140124-1

Ziglam, H. M., Baldwin, D. R., Daniels, I., Andrew, J. M., and Finch, R. G. (2002). Rifampicin concentrations in bronchial mucosa, epithelial lining fluid, alveolar macrophages and serum following a single $600 \mathrm{mg}$ oral dose in patients undergoing fibre-optic bronchoscopy. J. Antimicrob. Chemother. 50, 1011-1015. doi: $10.1093 / \mathrm{jac} / \mathrm{dkf2} 14$

Conflict of Interest Statement: The authors declare that the research was conducted in the absence of any commercial or financial relationships that could be construed as a potential conflict of interest.

Copyright (C) 2015 Dutta and Karakousis. This is an open-access article distributed under the terms of the Creative Commons Attribution License (CC BY). The use, distribution or reproduction in other forums is permitted, provided the original author(s) or licensor are credited and that the original publication in this journal is cited, in accordance with accepted academic practice. No use, distribution or reproduction is permitted which does not comply with these terms. 